\title{
Anestesia general con máscara laríngea para radioterapia infantil: técnica y complicaciones
}

\author{
General anesthesia with laryngeal mask airway \\ management for radiotherapy in children: anesthetic \\ technique and complications
}

DANIELA TOLEDO' ${ }^{1}$, BÁRBARA SILVA', MARCELA CÁRCAMO²

\begin{abstract}
Objective: Describe the use of general anesthesia with laryngeal mask airway management, and the presence of complications in children who require anesthesia for radiotherapy. The demographic and clinical characteristics of the children studied will also be described. Material and Methods: This is a descriptive, retrospective study. Clinical and demographic data were obtained from radiotherapy procedures with general anesthesia in children under 15 years of age completed between 2014 and 2016. The data collected included: sex, age, number of radiotherapies with general anesthesia, diagnosis, type of anesthesia, airway management device, complications (nausea and vomiting, laryngospasm and bronchopulmonary aspiration). The data were analyzed through descriptive statistics. Results: 1,285 radiotherapies where analyzed in 65 children. All patients received general anesthesia. Of the total sessions, only in 85 (7\%). Was required to install a peripheral intravenous line or the patient had one. In 1,139 (94\%) of the cases, the preferred airway management device was laryngeal mask, 64 (5\%) presented with tracheostomy, and in $8(0.66 \%)$ patients it was necessary to use an orotracheal tube. Nausea and vomiting occurred in 71 cases (5.53\%), and laryngospasm in only $9(0.7 \%)$ patients. Conclusion: General anesthesia with laryngeal mask airway management is a useful technique associated with low complications when caring for children requiring anesthesia for radiotherapy. Also, in this context, it seems unnecessary to routine install a peripheral intravenous line.
\end{abstract}

Unidad de Anestesia, Instituto Nacional del Cáncer, Chile.

Centro de Responsabilidad de Investigación, Instituto Nacional del Cáncer, Chile.

Fecha de recepción: 18 de febrero de 2018

Fecha de aceptación: 08 de marzo de 2018

ORCID

https://orcid.org/0000-0002-2916-0492

\section{Correspondencia:}

Daniela Toledo Arrau

Avenida -Profesor Zañartu 1010, Independencia

Teléfono móvil:+56 968376269 ,

Email: dtoledoarrau@gmail.com 


\section{RESUMEN}

Objetivo: El objetivo de este estudio es describir la técnica de anestesia general con máscara laríngea y la presencia de complicaciones anestésica en niños sometidos a radioterapia entre el 2014 y el 2016 en el Instituto Nacional del Cáncer. Secundariamente, se describirán las características demográficas y clínicas de la población estudiada. Material y Método: Estudio descriptivo, retrospectivo. Se extrajeron datos clínicos y demográficos de radioterapia bajo anestesia general realizados entre 2014 y el 2016 en niños menores de 15 años. Los datos recopilados fueron: edad, sexo, número de radioterapias con anestesia general, diagnóstico, tipo de anestesia, dispositivo de la vía aérea utilizado, complicaciones (náuseas, vómitos, laringoespasmo, aspiración broncopulmonar). El análisis estadístico fue descriptivo. Resultados: Se registraron 1.285 radioterapias en 65 niños. Todas con anestesia general. Del total de sesiones, en 85 (7\%) fue necesario instalar una vía venosa periférica o el paciente contaba con una. Con respecto a los dispositivos de vía aérea, en $1.139(94 \%)$ se utilizó máscara laríngea, en $64(5 \%)$ presentaba traqueostomía y en $8(0,66 \%)$ fue necesario la intubación orotraqueal. Dentro de las complicaciones encontramos náuseas y/o vómitos en 71 sesiones, correspondientes al 5,53\%. Espasmo laríngeo ocurrió en 9 (0,7\%) de las sesiones. Conclusiones: La anestesia general con máscara laríngea es una técnica útil y con pocas complicaciones para el manejo de niños que requieren radioterapia, donde parece no ser indispensable contar con una vía venosa periférica o central de rutina.

\section{Palabras clave:}

Anestesia general, radioterapia, pediatría, retrospectivo.

\section{Introducción}

S egún datos internacionales, la incidencia de cáncer en niños de 0-14 años entre el 2001 y 2010 fue de 140,6 por millón de personas año[1]. En Chile, constituye la segunda causa de muerte en menores de 15 años, con una incidencia entre 440 - 600 casos nuevos por año. Dentro los tipos más frecuentes encontramos las leucemias (40\%), los tumores relacionados al sistema nervioso central (17\%), linfomas $(13 \%)$ y tumores óseos (7\%). Los sarcomas de partes blandas, tumor de Wilms, retinoblastomas y tumores germinales representan un porcentaje menor[2].

Dentro de los tratamientos para el manejo del cáncer tanto en la población adulta como infantil, la radioterapia (RDT) es un pilar fundamental. En la población pediátrica, esta debe ser realizada en muchas oportunidades bajo anestesia general o sedación profunda para manejar el grado de inmovilidad y control de la ansiedad necesaria.

Al momento de tomar la decisión con respecto a la técnica anestésica a utilizar, junto con considerar los aspectos mencionados previamente, se debe tomar en cuenta las múltiples sesiones necesarias, los efectos deletéreos propios del tumor, la quimioterapia y la presencia de enfermedades concomitantes.
Y para complicar aún más el escenario, existe miedo, dolor, inmadurez emocional y estrés, no sólo en los niños, sino que también en sus familiares[3].

En la literatura existen diversos reportes sobre las técnicas anestésicas utilizadas para este procedimiento, que van desde ketamina[4,5] midazolam, hidrato de cloral, propofol[3,6] e incluso dexmedetomidina[7], manteniendo la ventilación espontánea con buenos resultados. Sin embargo, esto puede implicar punciones o uso del catéter venoso central con reservorio en forma reiterada, con las evidentes incomodidades, riesgo de infección y de provocar daño al catéter implantado[8].

Actualmente no existen datos latinoamericanos que describan la técnica anestésica utilizada, las características clínicas y la frecuencia de las complicaciones asociadas a la sedación o anestesia general en pacientes pediátricos sometidos a RDT.

Es por esto, que el objetivo de este estudio es describir la experiencia en el Instituto Nacional del Cáncer, en el uso de anestesia general con máscara laríngea y la presencia de complicaciones anestésicas en niños sometidos a radioterapia entre el 2014 y el 2016. Secundariamente, se describirán las características demográficas y clínicas de la población pediátrica en estudio. 


\section{Materiales y Métodos}

Estudio descriptivo, retrospectivo. Tras obtener la aprobación por el comité de ética local, se extrajeron datos clínicos y demográficos desde fuentes secundarias (libros de registros, hoja de anestesia y preanestesia), de los procedimientos de radioterapia bajo anestesia general realizados entre 2014 y el 2016 en niños menores de 15 años, con diagnóstico de cáncer de cualquier tipo o estadio. Sólo se incluyeron las sesiones de radioterapia, excluyendo aquellas destinadas a simulación. Los datos recopilados incluyen edad, sexo, número de RDT con anestesia general por niño, diagnóstico oncológico, tipo de anestesia general, dispositivo utilizado para el control de la vía aérea, ya sea máscara laríngea, traqueostomía o intubación orotraqueal, complicaciones durante la radioterapia y en la unidad de cuidados postanestésicos (náuseas, vómitos, laringoespasmo, aspiración broncopulmonar). El laringoespasmo se definió como tal, frente a la necesidad de ventilación con presión positiva o el uso de lidocaína intravenosa al momento de la inducción, mantención o emergencia de la anestesia general y, por su parte, se consideró sospecha de riesgo de aspiración broncopulmonar cuando se describe contenido gástrico en el tubo orotraqueal, máscara laríngea o cavidad oral tras la inducción o emergencia de la anestesia.

Otros antecedentes recopilados fueron: procedimientos realizados antes o después de la radioterapia como kinesioterapia, nebulizaciones, profilaxis náuseas y vómitos y quimioterapia previa el mismo día. También se registró la presencia de catéter venosos central con reservorio y/o vía venosa periférica, gastrostomía y sonda nasogástrica. Se incluyeron además datos relacionados con la duración de la anestesia y el tiempo desde la llagada del paciente a recuperación y la primera ingesta.

Se realizó un análisis estadístico descriptivo, se- gún tipo y escala de variable. El software utilizado fue STATA 13.

\section{Resultados}

Se registraron 1.285 sesiones de radioterapia, correspondiendo a 65 niños que iniciaron o continuaban su radioterapia desde agosto de 2014 hasta diciembre de 2016, siendo la última sesión registrada en febrero de 2017. No se incluyeron sesiones de simulación. No se logró tener acceso a los datos de 8 pacientes, ya que el registro no fue encontrado.

De los 65 niños estudiados, 731 (57\%) fueron hombres, y $554(43 \%)$ corresponden a mujeres, con una edad media de 1,28 años ( $D E \pm 0.56$ ).

El número de sesiones de radioterapia por niño varió entre 1 - 35, con una mediana de 23 .

La distribución de las frecuencias de diagnóstico y su participación en el número de sesiones de radioterapia se muestran en la Tabla 1.

Del total de sesiones, en 134 (10\%), los pacientes realizaban quimioterapia el mismo día previo a la sesión de radioterapia.

Dentro de los dispositivos invasivos que tenían los pacientes en cada una de sus sesiones, 686 contaban con un catéter venoso central (53\%) versus 497 (39\%) que no tenían. En 102 casos (8\%) no se pudo determinar si contaban o no con este dispositivo. En el caso de las gastrostomías, se presentaron en 98 (8\%) sesiones, sin gastrostomía en 1.161 (90\%) y sin información en 26 (2\%). La sonda nasogástrica se encontró en $175(14 \%)$ sesiones, ausencia de esta en $1.083(84 \%)$ y $\sin$ datos en $26(2 \%)$.

Cuando se analizó el tipo de anestesia, en todos los casos se realizó anestesia general. La inducción debió ser realizada con anestesia intravenosa a través de un catéter venoso central o vía periférica en 8 casos $(0,6 \%)$, en el resto de las 1.234 (96\%) fue con induc-

\begin{tabular}{lcccc}
\multicolumn{5}{c}{ Tabla 1. Distribución de frecuencias según diagnóstico } \\
\hline \multicolumn{1}{c}{ Diagnóstico } & $\mathbf{n}$ casos & $\begin{array}{c}\text { Porcentaje (\%) } \\
\text { casos }\end{array}$ & n sesiones & $\begin{array}{c}\text { Porcentaje (\%) } \\
\text { sesiones }\end{array}$ \\
Tumores SNC & 25 & 38 & 675 & 53 \\
Tumores de Wilms & 15 & 23 & 110 & 9 \\
Sarcomas & 11 & 17 & 260 & 20 \\
Linfomas, neuroblastomas & 3 & 5 & 81 & 6 \\
y retinoblastomas & & & 159 & 12 \\
Otros & 11 & 17 & 1.285 & 100 \\
Total & 65 & 100 & & \\
\hline
\end{tabular}


ción inhalatoria. En 42 (3\%) sesiones, no se obtuvo el dato. Con respecto a la mantención de la anestesia general, en un 97\% (1.242) fue con anestesia inhalatoria, en ninguno se documentó anestesia intravenosa y en el resto de los casos no se tuvo el antecedente específico (3\%).

Del total de sesiones, en 85 (7\%) fue necesario instalar una vía venosa periférica o el paciente contaba con una. No fue necesario en $1.161(90 \%)$, y no se contó con el antecedente en 39 (3\%).

Al analizar los datos con respecto a los dispositivos de vía aérea, se obtuvo 1.211 (94\%) registros del total. De éstos, en 1.139 (94\%) se utilizó máscara laríngea, en $64(5 \%)$ la traqueostomía y en $8(0,7 \%)$ fue necesario la intubación orotraqueal.

Dentro de las complicaciones, se encontraron náuseas y/o vómitos en 71 sesiones (6\% del total). Estos 71 casos de náuseas y vómitos ocurrieron en 35 niños, 21 de sexo femenino y 14 de sexo masculino. Se realizó profilaxis con ondansetrón sublingual en $218(17 \%)$ casos del total de sesiones. En los casos donde ocurrieron las náuseas y vómitos, se realizó profilaxis en 39 (55\%).

El espasmo laríngeo ocurrió en $9(0,7 \%)$ de las sesiones. Ninguno necesito intubación orotraqueal u hospitalización tras el evento.

En un caso hubo sospecha de aspiración broncopulmonar correspondiendo a una frecuencia de $0,08 \%$ del total.

Dentro de los requerimientos previo y después de la sesión de radioterapia, en 150 (12\%) sesiones fue necesario realizar kinesioterapia previa y en 12 (1\%), posterior a la sesión. En 142 (11\%) se realizaron nebulizaciones previas y en $10(0,78 \%)$ al terminar la sesión.

Al analizar 1.113 datos de tiempo de duración de anestesia, que corresponden al $87 \%$ del total de sesiones, el tiempo medio de anestesia fueron 24,55 minutos, ( $D E \pm 7,53$ minutos). La mediana del tiempo desde la llegada a recuperación hasta la primera ingesta, con 701 datos analizados (55\% del total de sesiones), es de 30 minutos (p25:30 minutos; p75: 40 min (min. 10, máx. 100).

\section{Discusión}

En este estudio se pudo observar que la anestesia general con máscara laríngea es una técnica anestésica útil y con pocas complicaciones en niños sometidos a radioterapia. Considerando que este es un procedimiento que va a ser repetido en múltiples oportunidades (hasta 30 veces) en forma diaria, resulta relevante analizar la necesidad de una vía venosa de rutina. Se analizan en forma separada la frecuencia de catéter venoso central y vía venosa periférica. Esto se debe a que la práctica habitual del centro estudiado es a evitar, en lo posible, el uso del catéter con reservorio, por lo que el requerir de una vía venosa periférica resulta ser un dato más adecuado para estimar la necesidad de administrar medicamentos o de la percepción por parte del anestesiólogo de contar con una vía venosa de respaldo para el procedimiento, siendo en este estudio considerado necesario sólo en el 7\% de los casos. A pesar de lo anterior, no se registró en las sesiones analizadas, la ocurrencia de algún evento adverso severo.

Es importante considerar que existen medidas organizacionales que podría participar en estos resultados, ya que existe un equipo de enfermería y auxiliares especialmente designadas para esto. Además, el paciente es evaluado previamente por un anestesiólogo quien define los requerimientos para llevar a cabo el procedimiento. Junto a lo anterior, se cuenta con unidades monitorizadas exclusivas para su cuidado, junto con la disponibilidad inmediata de asistencia por otros profesionales como kinesiólogos y oncólogo tratante. Por lo tanto, existe un trabajo en equipo multidisciplinario que permite entregar un cuidado seguro y dirigido a las características propias del paciente y del procedimiento.

Con respecto al manejo de la ansiedad por parte de los niños antes del procedimiento, no se realiza sedación optándose por el uso de medidas ambientales para su control, para lo cual se encuentra disponible una sala especialmente diseñada para este propósito. Esto resulta relevante por el alto número de sesiones al que tendrá que ser sometido el niño.

Dentro de las complicaciones más importantes se encontró el laringoespasmo con una prevalencia del $0,7 \%$ del total de las sesiones donde fue posible extraer el dato. Esto equivale a 9 sesiones en 5 niños, 4 de las veces ocurrió en la misma paciente. Esta paciente corresponde a una menor entre 2 y 3 años, con diagnóstico de neuroblastoma. No tiene antecedente de haber requerido kinesioterapia o nebulizaciones previas a los procedimientos, por lo que pareciera ser una característica especial de la paciente, ya que no se observa la presencia de factores de riesgo clásicos. Ninguno requirió intubación orotraqueal o cuidados especiales posteriores al procedimiento. Por lo tanto, a pesar de existir intervención reiterada de la vía aérea, el laringoespasmo no parece ser una complicación frecuente, aunque siempre se debe estar atento a su eventual aparición.

La necesidad de intubación orotraqueal fue ne- 
cesaria en 8 oportunidades, no se obtuvieron datos sobre si fue esperada o inesperada, sólo en un solo caso, de sexo femenino, con diagnóstico de un tumor del sistema nervioso central, fue necesario cambiar en 4 sesiones desde máscara laríngea a intubación orotraqueal. Esto resulta curioso, ya que, en muchos casos, al ser necesario irradiar la zona de la cabeza, al cambiar el dispositivo de vía aérea hay que volver a realizar simulación con el nuevo dispositivo. Destaca en este caso la necesidad de kinesioterapia y nebulización previa a las sesiones en 10 oportunidades, instalación de vía venosa periférica en 10, y luego la instalación de catéter venoso central a partir de la sesión número 9 de un total de 31, por lo que podemos sospechar que se trata de un caso más complejo que lo habitual.

La sospecha de aspiración broncopulmonar fue muy baja, con sólo un caso. Este caso corresponde a una paciente entre 2 a 3 años, con diagnóstico de tumor del sistema nervioso central, la cual no habría cumplido con el ayuno según datos entregados por la madre. La sospecha de aspiración broncopulmonar se debe a la presencia de leche en la máscara laríngea al momento de su retiro. La paciente, luego del procedimiento, es monitorizada en la unidad de cuidados postanestésicos. No se evidencian complicaciones adicionales durante su estadía y es dada de alta con las indicaciones habituales.

En un estudio similar [9] donde se estudiaron 1.033 procedimientos de radioterapia bajo sedación, donde 833 fueron realizados con anestesia general, no se reportaron complicaciones en el $97 \%$ de los casos, sin embargo, el análisis se hace considerando tanto la sedación consciente como la anestesia general. Por otra parte, en este mismo estudio, sólo un $4 \%$ no contaba con un acceso venoso, y en aquellos en los que se contaba, este era utilizado en forma frecuente en la medida en que avanzaba el número de radioterapias en el paciente. Incluso se reporta que el $93 \%$ de los procedimientos con acceso venoso eran calificados como satisfactorios cuando se contaba con un acceso venoso, en contraste con el $73 \%$ reportado como satisfactorio cuando no se contaba con este.

Por último, se obtuvieron también datos relevantes al momento de gestionar los tiempos y recursos de una unidad destinada a proveer RDT infantil bajo anestesia. Dentro de los procedimientos antes y después de la RDT, lo más frecuente es la kinesioterapia por lo que resulta importante contar con este recurso. Con respecto a los tiempos, y de acuerdo con los datos obtenidos se deberían programar por paciente aproximadamente 30 min, aunque este valor va a depender del diagnóstico, tipo de sesión (ya que en algunos momentos se requiere realizar cálculos y estudios adicionales), la necesidad de toma de muestras sanguíneas, etc.

Este estudio constituye una mirada global de la anestesia para radioterapia infantil. Una limitante importante es la falta de datos completos en 8 pacientes. Parece relevante realizar estudios adicionales sobre seguridad, poniendo énfasis en la creación de bases de datos y en el registro apropiado de los antecedentes de las sesiones, ya que como se mencionó en los resultados no obtuvimos datos en algunas de las áreas a evaluar.

Con los antecedentes obtenidos resulta al menos osado entregar alguna recomendación con respecto al manejo de estos pacientes, ya que se mencionó previamente, la baja tasa de complicaciones posiblemente esté relacionada con elementos organizacionales, que hacen posible realizar este procedimiento bajo las condiciones descritas. Futuros desafíos están en determinar factores de riesgo para náuseas y vómitos y la utilidad de la profilaxis, así como establecer asociaciones entre los diagnósticos y requerimientos clínicos y organizacionales, para de esta forma mejorar la eficacia y eficiencia del procedimiento.

\section{Referencias}

1. International incidence of childhood cancer, 2001-10: a population-based registry study - The Lancet Oncology [Internet]. [citado 12 de junio de 2017]. Disponible en: http://www. thelancet.com/journals/lanond article/PIIS1470-2045(17)301869/fulltext
2. (Microsoft Word - GPC Linfoma y tumor s\363lido en menores de 15 al361os. 22.11.09. doc) - 7220fdc433ed44a9e04001011f0113b9.pdf [Internet]. [citado 12 de junio de 2017]. Disponible en: http://web. minsal.cl/portal/url/item/7220fdc 433ed44a9e04001011f0113b9. pdf

3. McFadyen JG, Pelly N, Orr RJ.
Sedation and anesthesia for the pediatric patient undergoing radiation therapy. Curr Opin Anaesthesiol. agosto de 2011;24(4):433-8.

4. Soyannwo OA, Amanor-Boadu SD, Adenipekun A, Sanusi AA, Akinyemi OA. Ketamine anaesthesia for young children undergoing radiotherapy. West Afr J Med. junio de 
2001;20(2):136-9.

5. Shewale S, Saxena A, Trikha A, Singh $M$, Sharief A. Oral ketamine for radiotherapy in children with cancer. Indian J Pediatr. abril de 2000;67(4):263-6.

6. Owusu-Agyemang P, Grosshans D, Arunkumar R, Rebello E, Popovich S, Zavala A, et al. Noninvasive anesthesia for children undergoing proton radiation therapy. Radiother Oncol. 1 de abril de 2014;111(1):30-4.

7. Shukry M, Ramadhyani U. Dexmedetomidine as the primary sedative agent for brain radiation therapy in a 21-month old child. Paediatr Anaesth. marzo de 2005;15(3):241-2.

8. Adenipekun A, Soyannwo OA, Amanor-Boadu SD, Campbell OB, Oyesegun AR. Complications following sedation of paediatric oncology patients undergoing radiotherapy. West Afr J Med. diciembre de 1998;17(4):224-6. 9. Seiler G, De Vol E, Khafaga Y, Gregory B, Al-Shabanah M, Valmores $A$, et al. Evaluation of the safety and efficacy of repeated sedations for the radiotherapy of young children with cancer: a prospective study of 1033 consecutive sedations. Int J Radiat Oncol Biol Phys. 1 de marzo de 2001;49(3):771-83. 